\title{
Open Letter to the Internet Mathematics Community
}

\author{
Anthony Bonato and Fan Chung Graham \\ Editors-in-Chief
}

The World Wide Web and Internet have evolved rapidly in the last decade. The web currently contains over a trillion webpages, with an increasingly diverse range of content such as images, blogs, audio, and video. Web search, the economic theory of network formation, and generative network models are just a few of the active research areas surrounding the web and Internet.

Complex networks in general remain a hot topic in many fields. We are now witnessing the explosive growth and popularity of online social networks such as Facebook, LinkedIn, and Twitter, where an estimated half of people who access the Internet are registered users. New discoveries in biology are leading to an increased integration of a systems approach, where networks of protein-protein and other known physical interactions are studied and modeled.

As new discoveries emerge in the field of complex networks, researchers are faced with enormous new challenges in their mining and modeling. Apart from the complexities surrounding their large-scale nature, complex networks often require a hybrid approach, merging the fields of mathematics with computer science, physics, biology, sociology, and economics.

In keeping with the rapid evolution of the field of complex networks, Internet Mathematics - which is the leading reference for fast-breaking research in the field - is itself changing. First, we welcome several new additions to our editorial board. Anthony Bonato is joining the journal as co-editor-in-chief. The editorial board and managing editors are also changing, with the addition of highly respected researchers such as Jennifer Chayes, Rick Durrett, and Andrew Tomkins to the board.

Second, the scope of the journal is also broadening. In addition to more mathematically oriented papers, the journal will accept experimental and empirical works reporting on important new discoveries. High-quality papers from all areas 
of complex networks will be accepted, such as those on the web graph, Internet graph, online social networks, wireless networks, and biological networks. Special issues will continue for selected full papers from proceedings of leading conferences such as WAW, WINE, and WSDM.

As we face the future, the journal needs the continued support of the Internet mathematics complex network community. We encourage new subscriptions from universities, colleges, and institutions in the private sector and beyond with an interest in research on networks. We also encourage researchers to continue sending us their high-quality papers. With a renewed effort to reduce our backlog and expedite the review process, we are confident that the journal will remain as the principal reference for fast-breaking topics in the field.

The future has no doubt many exciting discoveries waiting for us in network science. Internet Mathematics is, and has been, an outstanding showcase for such discoveries since its inception seven years ago. We are confident that with the energy brought by the new board members and the journal's renewed scope, Internet Mathematics will remain a leading reference for network science in the years to come. 